\title{
Le statut a-t-il un genre? La perception du français et de l'anglais à Montréal en 1960 et en 2004
}

\author{
Elke Laur \\ Office québécois de la langue française \\ elaur@oqlf.gouv.qc.ca
}

\section{Introduction}

Nous savons par maintes recherches que les femmes et les hommes ont des comportements différents. Peu importe si ces différences sont enracinées à un niveau neurologique (Kimura, 1999) ou social, elles se reflètent dans le comportement linguistique. Plusieurs langues présentent en effet des distinctions phonétiques ou morphologiques liées au genre des locuteurs ou locutrices. Mais au delà de ces distinctions langagières, maintes différences sociolinguistiques liées au genre peuvent être observées au sein d'une communauté : le style conversationnel, la prononciation, le rapport à la norme (Trudgill, 1972; Labov, 1990; Armstrong et autres, 2001 entre autres).

Il n'est pas étonnant que ces différences comportementales semblent se refléter également dans la perception linguistique. La voix inégale de l'homme et de la femme, due à la taille différente des cordes vocales chez l'adulte, produisent une hauteur divergente. Cette divergence est audible et peut donc influencer les interlocuteurs. Mais cette différence physiologique ne semble pas être la seule cause d'une éventuelle différenciation perceptive, puisque même les voix d'enfants prépubères sont distinguées selon leur sexe par une majorité d'adultes (Sachs, Lieberman et Erickson, 1973). Il est entre autres supposé que l'intonation ou les fréquences plus hautes ou plus basses de la prononciation de certaines voyelles seraient intégrées dans un schème inconscient et sous-jacent à chaque genre (Labelle).

Les études sur la perception des langues par contre ne tiennent que peu ou pas du tout compte de ces différences dues au genre. Dans cet article, nous allons présenter une méthodologie-type de la mesure des attitudes et des perceptions linguistiques afin de démontrer l'importance d'une prise en compte du genre dans l'étude de l'évaluation des langues ou des variétés linguistiques.

\subsection{Le principe de la méthodologie des faux-couples et son contexte historique}

Il y a plus de 40 ans, une équipe de psychologues sociaux de l'Université McGill à Montréal a inventé une nouvelle méthodologie afin de pouvoir étudier les évaluations linguistiques et d'en déduire l'attrait qu'une langue exerce sur les locuteurs. Cet accès indirect aux perceptions linguistiques se fait par l'étude des associations que font les gens quand ils entendent le français ou l'anglais. Le côté original de cette méthodologie réside dans une astuce, un tour de prestidigitation : les sujets entendent plusieurs fois la voix d'un même locuteur qui parle avec plusieurs accents, langues, niveaux langagiers, etc., mais ont l'impression qu'il s'agit de personnes différentes. Ainsi, alors qu'ils pensent évaluer les différentes qualités esthétiques des voix qu'ils entendent, les sujets jugent en fait d'autres dimensions qui ne sont pas évoquées par la personnalité du locuteur, mais par l'accent, la langue ou le niveau de langue. Cette méthodologie reste encore aujourd'hui une des plus ingénieuses et efficaces puisqu'elle permet de déjouer une certaine autocensure dans les réponses des personnes.

En 1959, une soixantaine d'étudiants en première année à l'université McGill de Montréal ainsi qu'une soixantaine de collégiens d'un collège catholique francophone ont eu à évaluer des voix en anglais et en français (entre autres tâches) sur 14 traits de caractère. Selon les auteurs, il n'y avait aucun indice permettant de croire que ces étudiants se sont aperçu que les voix qu'ils évaluaient appartenaient à trois hommes parfaitement bilingues qui parlaient à tour de rôle en français et en anglais (1960:44). Cette méthodologie visait à apparier (ou à coupler - d'où le nom de faux-couples) deux façons de parler (deux langues ou deux variétés de langue) en utilisant des personnes bilingues (ou bidialectales) afin de 
connaître la perception de ces parlers, a fait le tour du monde depuis. Elle est devenue la méthode de prédilection pour accéder indirectement aux jugements linguistiques et figure des centaines de fois dans le Social Science Index. Les voix utilisées pour faire évaluer le français et l'anglais à Montréal étaient exclusivement des voix masculines - tout comme dans des études qui ont reproduit cette recherche dans le même contexte par la suite (voir surtout Genessee et Holobow, 1989). Pourtant, en 1963 déjà, un étudiant de maîtrise de W.E. Lambert à l'Université McGill constate une certaine incidence du sexe des locuteurs sur les perceptions (Preston, 1963). Ainsi, tout en conservant l'idée originale de l'étude - celle de faire évaluer des langues à travers la perception de locuteurs bilingues - nous avons dans notre recherche changé quelques aspects méthodologiques afin de tester et d'améliorer les résultats.

\subsection{Méthodologie comparative}

Les aspects méthodologiques que nous avons changés par rapport à celle de Lambert et de ses collègues concernent les deux aspects qui sont le plus souvent objets de variation dans les études utilisant cette méthode: les voix et les juges. Ainsi, au lieu d'utiliser exclusivement des voix masculines, nous avons enregistré les voix d'une femme et d'un homme bilingues afin de vérifier l'influence du sexe des voix sur l'évaluation ${ }^{1}$. Le questionnaire comporte des échelles d'évaluation de type Likert (très, assez, peu et pas $d u$ tout, en français et very, somewhat, not very, not at all en anglais) pour 12 caractéristiques (choisies et traduites par analogie avec l'étude originale ainsi qu'avec d'autres études faites selon cette même formule): être sympathique (likeable), sociable (sociable), intelligent (intelligent), distingué (distinguished), chaleureux (warm), dynamique (dynamic), instruit (educated), fiable (dependable) et avoir les qualités d'un leader (having the qualities of a leader), le sens de l'humour (having a sense of humour), du caractère (having character) et de l'ambition (having ambition). La version anglaise et la version française du questionnaire ont été utilisées selon la préférence des interlocuteurs.

En ce qui concerne les juges, nous avons élargi leur nombre à 610 personnes. Stratifié selon les langues maternelles, cet échantillon est représentatif de la population de l'île de Montréal $^{2}$ et inclut donc des personnes de tous les groupes d'âge, des deux sexes ainsi que de différents quartiers de l'île de Montréal. Cette variation semble importante, puisqu'une recherche dans une compilation de résumés scientifiques à partir de plus de 2000 revues dans le domaine de l'étude des langues et du comportement (LLBA, Linguistics and Language Behaviour Abstracts, accessibles sur le site http://ccat.sas.upenn.edu/ haroldfs/bibliogs/MACHGUIS. HTM) nous enseigne que la technique des faux-couples a toujours été administrée à de petits groupes de personnes, souvent des étudiants. Grâce aux nouvelles technologies de l'information qui permettent de faire entendre des voix préenregistrées à un grand nombre de personnes, tout en recourant à la technique de l'enquête téléphonique, nous avons pu élargir l'échantillon. Normalement utilisée pour l'élicitation d'évaluations plutôt directes, la dialectologie semble également utiliser ces nouvelles possibilités technologiques de sondage (Labov, Ash et Boberg, 2004). Mais en ce qui concerne la méthodologie des faux-couples, il s'agit, à notre connaissance, de la première étude qui l'utilise auprès d'un échantillon représentatif. Une pondération de l'échantillon constitué avec des strates non proportionnelles nous permet en outre de faire des inférences statistiques sur la population cible, c'est-à-dire la population de l'île de Montréal. L'influence de ces différentes caractéristiques des juges sur les évaluations sera examinée en détail ailleurs (Laur, à paraître). Nous allons nous concentrer ici simplement sur l'influence que l'utilisation de la voix de femme a pu produire comparativement - sur les évaluations du français et de l'anglais à Montréal.

\section{La perception hétéroclite}

Comme Calvet (1999) l'affirme, il est vrai que les personnes, en général, confondent la langue avec le locuteur qui la parle. Les Montréalais n'ont eu aucun problème à attribuer une cote aux voix qu'ils entendaient et à évaluer plus ou moins fortement les caractéristiques présentées. De plus, les Montréalais ont des opinions assez distinctes quant aux voix qu'ils entendent: ils ont utilisé tous les points de l'échelle des évaluations qui leur était proposée, la variance pour chaque caractéristique se situe entre 0,34 et 0,94 points, la moyenne varie entre 2,2 et 3,2 ("peu » et « assez ») et l'écart-type varie entre 0,61 et 0,97 . Il s'avère donc que les évaluations varient d'une manière notable et que les perceptions 
linguistiques des Montréalais fluctuent quant à la notation et à l'appréciation qu'ils ont des différentes voix enregistrées. Dans un premier temps, nous avons cherché à savoir si les fluctuations dans les évaluations se regroupent entre elles d'une façon significative.

\subsection{La perception du statut linguistique}

Afin de connaître comment ces évaluations se regroupent, nous avons effectué des analyses factorielles exploratoires de l'ensemble des données. Deux facteurs sous-jacents expliquent $63 \%$ de la variance dans l'évaluation des caractéristiques :

Tableau 1 : Les solutions factorielles pour l'ensemble des caractéristiques évaluées

\begin{tabular}{|c|c|c|c|}
\hline Facteur & Caractéristiques & Saturations factorielles & $\boldsymbol{\alpha}$ de Cronbach \\
\hline \multirow{4}{*}{1} & sociable &, 879 &, 8705 \\
\cline { 2 - 4 } & chaleureux &, 856 & \\
\cline { 2 - 4 } & sympathique &, 806 & \\
\cline { 2 - 4 } & sens de l'humour &, 522 & \\
\cline { 2 - 4 } & distingué &, 438 &, 8685 \\
\cline { 2 - 4 } & dynamique &, 400 & \\
\hline \multirow{2}{*}{} & ambition &, 884 & \\
\cline { 2 - 4 } & instruit &, 706 & \\
\cline { 2 - 4 } & qualités d'un leader &, 696 & \\
\cline { 2 - 4 } & intelligent &, 593 & \\
\cline { 2 - 4 } & fiable &, 555 & \\
\cline { 2 - 4 } & caractère &, 548 & \\
\hline
\end{tabular}

Analyse factorielle avec extraction « alpha »; rotation oblique OBLIMIN, KMO = ,923; Eigenvalue > 1,0

Ces deux facteurs ressemblent beaucoup aux dimensions de statut et de solidarité décrites dans d'autres études (par exemple Giles et Ryan, 1982; Zahn et Hopper, 1985; Genesee et Holobow, 1989; Dixon, Maltoney et Cocks, 2002). La dimension statut reflète un statut social relatif ou le pouvoir d'un groupe de locuteurs. La dimension solidarité fait par contre plutôt référence à une solidarité intragroupe et à une loyauté linguistique, c'est-à-dire un attrait social intracommunautaire. Le fait que l'ensemble des voix (parlant en anglais tout comme en français) se différencie selon ces dimensions montre que ces dimensions se trouvent sous-jacentes à la perception des langues en général (au moins les deux examinées) et que ces dimensions ne sont pas seulement propres à une (des deux) langue(s) en particulier. Ainsi, les facteurs identifiés se composent d'un côté de caractéristiques représentant le statut (avoir de l'ambition, du caractère, les qualités d'un leader, être instruit, intelligent et fiable) et de l'autre côté les caractéristiques associées à la solidarité (être sympathique, sociable, chaleureux, distingué, dynamique et avoir le sens de l'humour). Les saturations factorielles diffèrent, mais le facteur se confirme par le test de fiabilité.

Par contre, si on divise l'échantillon selon les langues utilisées, le résultat de l'analyse factorielle n'est plus le même. Nous savons depuis Osgood (1964) que le regroupement de caractéristiques, voire le classement en général de ces caractéristiques, est culturellement variable. D'ailleurs, dans les études utilisant la méthodologie des faux-couples, les dimensions sont constamment ajustées à cause d'une certaine différence dans la composition de ces dimensions selon les langues (Dixon, Maltoney et Cocks, 2002 pour l'anglais et le portugais ainsi que Genessee et Holobow, 1989, pour l'anglais et le français). Une telle différence dans le regroupement des caractéristiques pour chacune des langues évaluées se retrouve aussi dans les données que nous avons analysées. Les facteurs sous-jacents varient aussi dans nos données selon la langue entendue par les répondants - ou, plutôt, ce sont les caractéristiques formant les facteurs statut et solidarité qui varient selon les langues parlées par les locuteurs. Selon ces résultats, l'anglais est perçu en deux dimensions, tandis que la perception du français se décompose en trois facteurs (voir le tableau 2). Par conséquent, la perception des deux langues montre un nombre de facteurs sous-jacents différent selon la langue de la voix entendue. 
Les facteurs solidarité et statut se composent donc de caractéristiques différentes selon les souséchantillons considérés: (1) celui pour l'anglais, (2) celui pour le français et (3) celui de l'ensemble. Comparons les résultats pour ces trois sous-ensembles selon les facteurs sous-jacents. Considérons d'abord le facteur statut : ce ne sont que les traits fiable, intelligent et instruit qui contribuent au même facteur pour les trois groupes de données. Pour l'ensemble, tout comme pour les voix en anglais, six variables (intelligent, instruit, fiable, qualités d'un leader, caractère, ambition) forment le facteur statut, tandis que l'évaluation des voix en français est constituée de deux facteurs distincts pour ces caractéristiques : l'un qu'on pourrait nommer un facteur de «connaissance-culture», formé par intelligent, instruit, fiable, distingué, et l'autre, qui fait davantage appel à des valeurs de «statut», formé par caractère, leadership, ambition et dynamisme, qu'on pourrait nommer «compétence-action».

Tableau 2 : Les solutions factorielles pour les caractéristiques évaluées selon la langue

\begin{tabular}{|c|c|c|c|c|c|}
\hline \multirow{2}{*}{$\begin{array}{l}\text { Fac- } \\
\text { teur }\end{array}$} & \multicolumn{2}{|c|}{ Les voix en français KMO ,908 } & \multicolumn{2}{|c|}{ Les voix en anglais KMO ,907 } & \multirow{2}{*}{$\begin{array}{l}\text { Fac- } \\
\text { teur }\end{array}$} \\
\hline & Caractéristiques & Solutions factorielles & Caractéristiques & Solutions factorielles & \\
\hline \multirow[t]{8}{*}{3} & \multicolumn{2}{|c|}{$9 \%: \alpha$ de Cronbach : ,8050 } & \multicolumn{2}{|c|}{$47 \%: \alpha$ de Cronbach $:, 8480$} & 1 \\
\hline & intelligent & ,771 & intelligent & ,493 & \\
\hline & instruit &, 721 & instruit & ,616 & \\
\hline & fiable & ,471 & fiable & ,356 & \\
\hline & distingué & ,559 & $\begin{array}{l}\text { qualités d'un } \\
\text { leader }\end{array}$ & ,673 & \\
\hline & & & caractère & ,700 & \\
\hline & & & ambition &, 821 & \\
\hline & & & dynamique & ,382 & \\
\hline \multirow[t]{6}{*}{2} & \multicolumn{2}{|c|}{$11 \%: \alpha$ de Cronbach : ,8492 } & \multicolumn{2}{|c|}{$10 \%: \alpha$ de Cronbach :,8336 } & 2 \\
\hline & chaleureux & $\begin{array}{r}, 813 \\
\end{array}$ & chaleureux &, 905 & \\
\hline & sociable & ,770 & sociable & ,729 & \\
\hline & sympathique & ,688 & sympathique & ,806 & \\
\hline & sens de l'humour & ,611 & sens de l'humour & ,423 & \\
\hline & & & distingué & ,364 & \\
\hline \multirow[t]{5}{*}{1} & \multicolumn{2}{|c|}{$46 \%: \alpha$ de Cronbach : ,7961 } & & & \\
\hline & ambition &, 752 & & & \\
\hline & caractère & ,625 & & & \\
\hline & $\begin{array}{l}\text { qualités d'un } \\
\text { leader }\end{array}$ & ,603 & & & \\
\hline & dynamique & ,411 & & & \\
\hline
\end{tabular}

Une autre différence entre les sous-échantillons concerne l'importance des facteurs dans l'explication de la variance : pour l'anglais, c'est le facteur statut qui explique $47 \%$ de la variance. Les évaluations du français par contre s'expliquent avant tout avec le facteur compétence-action (46\%). Les traits qui forment le facteur solidarité par contre semblent covarier davantage pour l'ensemble des voix ainsi que pour les voix en français et en anglais séparément, puisque quatre caractéristiques en font partie dans les trois analyses: sympathique, sociable, chaleureux, sens de l'humour. Dans les trois cas, ce facteur explique à peu près $10 \%$ de la variance.

Ainsi, nous pouvons affirmer que l'évaluation du français et de l'anglais révèle différents facteurs sousjacents et que cette différence vaut pour tous les Montréalais. Le français est jugé en vertu de trois catégories que nous avons nommées statut, solidarité et compétence, tandis que l'évaluation de l'anglais se regroupe dans les deux premières. Il devient clair que la perception des langues est régie par des associations que tous les Montréalais partagent. Ces associations se basent sur des combinaisons de qualificatifs distinctes pour les deux langues.

En termes d'évaluation, les facteurs obtenus n'ont pas les mêmes moyennes non plus. Nous avons considéré les facteurs constitués à partir de l'ensemble des évaluations (voir le tableau 1, illustrés dans le 
graphique 1a) tout comme les facteurs constitués selon la répartition décrite dans le tableau 2 pour les voix en français et en anglais (illustrés dans le graphique 1b). Remarquons qu'en général, le facteur statut (constitué des caractéristiques intelligent, instruit, fiable) obtient une moyenne plus élevée que le facteur solidarité (composé des caractéristiques sympathique, sociable, chaleureux et le sens de l'humour) et le facteur compétence-action (dynamique, qualités d'un leader, caractère, ambition).

Les caractéristiques associées à un statut linguistique obtiennent donc généralement des moyennes plus élevées que les caractéristiques faisant plutôt référence à la solidarité. Ce constat s'applique également aux voix en français et aux voix en anglais comme le montre la partie b du graphique 1, même si les voix en anglais obtiennent pour les trois facteurs des moyennes plus élevées en comparaison des voix en français.

Graphique 1 : Les moyennes des facteurs solidarité, statut et compétence-action a. selon le tableau $1 \quad$ b. selon le tableau 2 et les deux langues
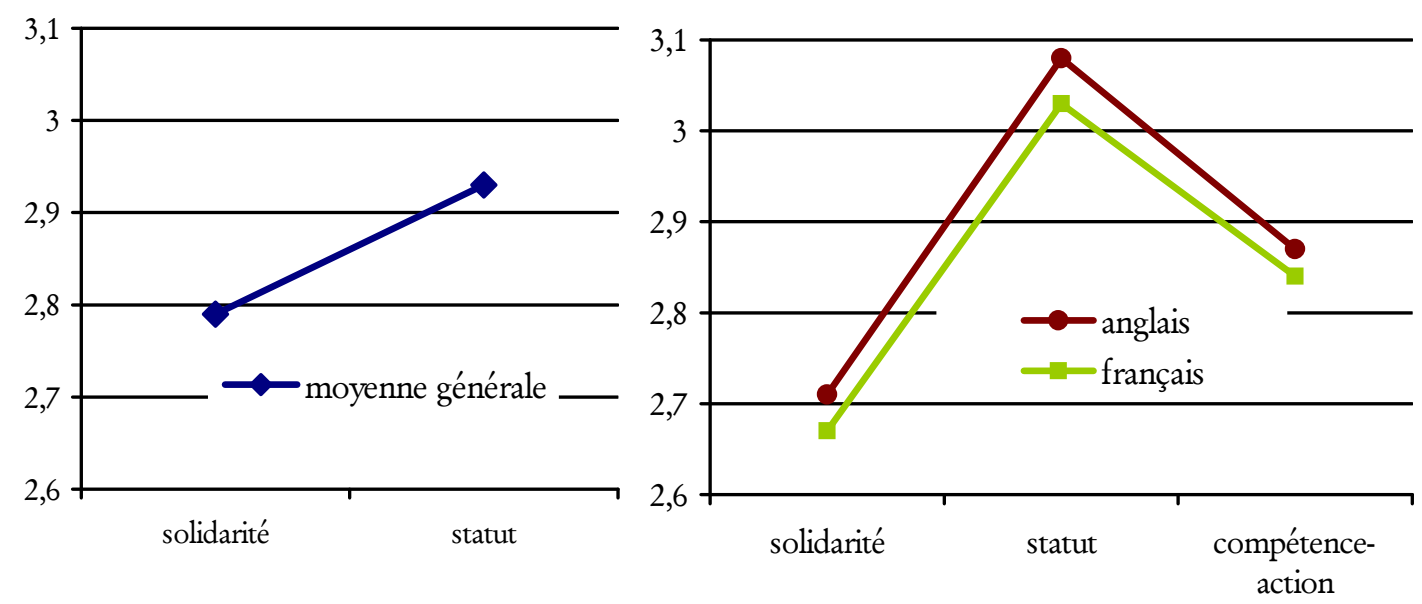

\subsection{Le statut, a-t-il un genre?}

Afin de savoir si une des caractéristiques ou un facteur est différemment évalué selon le genre des locuteurs, nous avons également procédé à des analyses factorielles en séparant, cette fois-ci, les évaluations des voix selon le sexe plutôt que selon la langue et il s'avère que les évaluations des voix de l'homme ressemblent dans les solutions factorielles aux évaluations des voix en anglais. Cette ressemblance nous a amené à vérifier si les données présentent une interaction entre le sexe et la langue des locuteurs. C'est l'analyse MANOVA pour mesures répétées qui peut nous donner plus de détails sur l'évaluation des langues et des genres et de leur interaction, autrement dit, si la voix de la femme (ou de l'homme) est significativement liée à l'une ou l'autre langue. Selon les résultats ${ }^{3}$, il y a effectivement une interaction significative pour tous les traits, sauf pour la caractéristique sens de l'humour. Le fait que ce soit une femme ou un homme qui parle français ou anglais a donc son importance. La variance expliquée par cette interaction dans les évaluations analysées n'atteint par contre que de $1 \%$ à $6 \%$ (il s'agit de l'importance de l'effet de l'interaction sur la variation des évaluations).

Ce résultat est particulièrement important - vu que la majorité des études montréalaises sur la perception (faites avec la méthodologie des faux-couples) semble en faire complètement abstraction : ce sont pour la grande majorité des études qui font évaluer exclusivement des voix d'homme. Or, ce résultat nous indiquerait qu'une étude sur la perception des langues ne peut pas ignorer le sexe des locuteurs dont témoignent leurs voix sans être partielle dans ses conclusions, voire induire des interprétations erronées. 
Puisque l'évaluation des langues et l'évaluation du genre des voix sont interreliées, deux perspectives sont possibles: l'analyse des résultats selon la langue des locuteurs et l'analyse selon le sexe des locuteurs. L'interaction reste évidemment la même, seul l'angle d'analyse diffère.

\subsection{1. Évaluation des caractéristiques selon la langue des locuteurs}

Pour identifier et comprendre ces différentes évaluations des langues, nous avons donc décomposé l'analyse en précisant l'effet de la langue dans l'interaction. Cette analyse nous permet de constater que les moyennes obtenues par la femme - aussi bien pour l'anglais que pour le français - sont supérieures à celles de l'homme sauf dans quatre cas : les caractéristiques caractère, dynamique, qualités d'un leader et ambition. Ces quatre traits obtiennent une meilleure moyenne lorsque c'est l'homme qui parle, mais en anglais seulement. Les résultats indiquent qu'à chaque niveau linguistique (pour l'anglais et pour le français), l'effet diffère significativement selon le sexe du locuteur. Par exemple, pour la caractéristique chaleureux, l'évaluation de la voix en anglais est plus élevée que celle en français lorsque le locuteur est un homme (voir le graphique ci-dessous), alors que l'évaluation de la voix en anglais est moins élevée que celle de la voix en français lorsque la voix du locuteur est une femme.

\section{Graphique 2 : Moyennes pour le trait chaleureux}

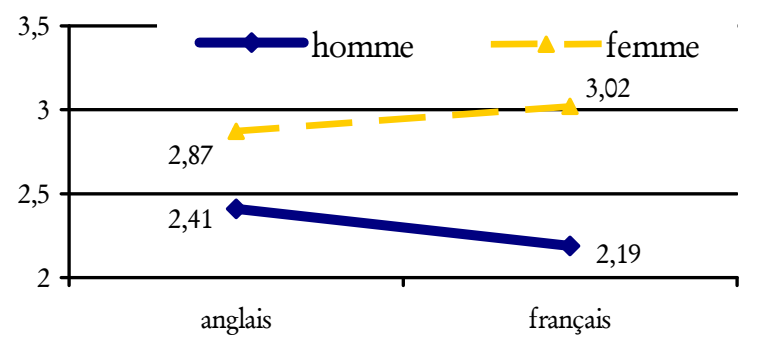

En d'autres mots, l'évaluation de la voix de l'homme baisse lorsqu'il parle en français par rapport à l'anglais, tandis que l'évaluation de la voix de la femme monte lorsqu'elle parle en français plutôt qu'en anglais. La différence entre les sexes est plus grande pour l'évaluation du français que pour celle de l'anglais $(0,83$ point de différence pour le français en comparaison de 0,46 point de différence pour l'anglais). L'interaction entre le sexe et la langue des voix existe donc puisque la différence des évaluations de l'anglais et du français n'est pas la même pour l'homme et pour la femme. Les évaluations diffèrent également pour les facteurs statut et solidarité. Le graphique suivant montre leurs distributions. Il illustre que les moyennes pour le facteur statut sont les plus élevées pour les deux sexes et pour les deux langues. Les moyennes pour le facteur solidarité sont généralement les plus basses - ce qui est particulièrement le cas pour la voix de l'homme, en français comme en anglais.

Graphique 3 : Moyennes des évaluations par enregistrement pour les facteurs

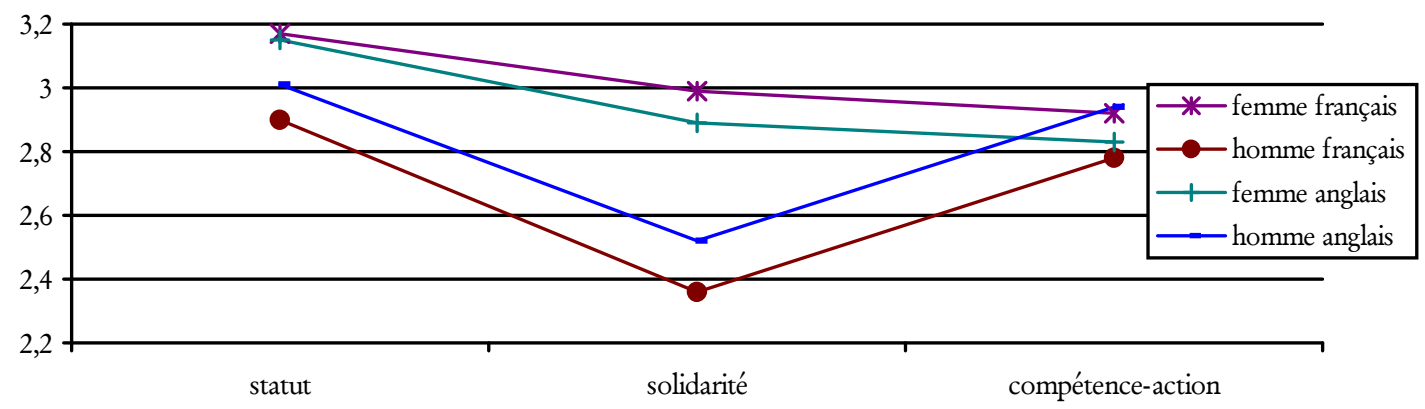


Le facteur compétence-action par contre inverse le rapport des langues et des sexes comparativement aux autres facteurs : les deux voix de la femme obtiennent des moyennes inférieures (2,92 en français et 2,83 en anglais) à la voix masculine - lorsqu'il parle en anglais (moyenne de 2,94). Dans le graphique suivant est illustré le facteur compétence-action.

\section{Graphique 4 : Moyennes pour le facteur compétence-action}

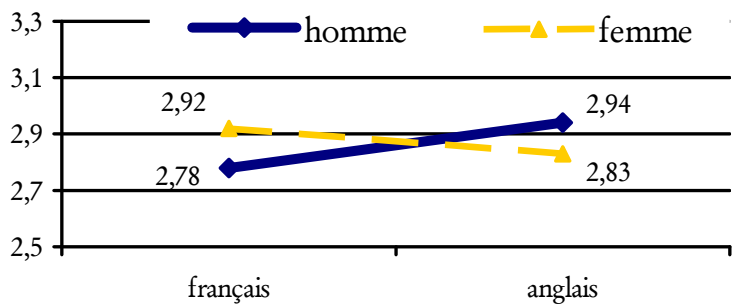

Le rapport des voix en français et en anglais est le même que pour les autres facteurs et caractéristiques (c'est-à-dire que la voix masculine est mieux évaluée lorsqu'elle parle en anglais plutôt qu'en français, tandis que la voix féminine est mieux évaluée lorsqu'elle parle en français plutôt qu'en anglais) - mais, en plus, la voix masculine en anglais obtient la moyenne la plus élevée de toutes les évaluations pour ce facteur, ce qui inverse le résultat des autres facteurs ou caractéristiques. Pour ce facteur, il y a donc davantage d'association entre l'anglais, l'homme et les caractéristiques regroupées par le facteur compétence-action. On peut donc dire que la valorisation de l'anglais est plus forte chez l'homme tandis que la valorisation du français est plus forte chez la femme. Le rapport des langues et du sexe est inversé. C'est-à-dire, qu'en moyenne, une personne va être perçue comme ayant davantage de statut, de solidarité et surtout de compétence si elle nous parle en anglais - mais seulement s'il s'agit d'un homme.

\subsection{2. Évaluation des caractéristiques selon le sexe des locuteurs}

Toutes les caractéristiques ont une moyenne un peu plus élevée pour l'évaluation de la voix féminine sauf pour la caractéristique ambition. Par contre, les écarts-types et la variance sont plus élevés pour les deux voix masculines - ce qui laisse penser à une plus grande différence entre les évaluations de l'anglais et du français parlé par l'homme par rapport à la femme. Ainsi, la voix d'une femme obtient - en moyenne - une évaluation plus élevée pour presque tous les traits. La voix d'une femme est - d'une façon générale - perçue plus positivement que la voix d'un homme.

Le graphique suivant illustre pour toutes les caractéristiques que la différence des moyennes entre les évaluations de la femme et de l'homme est plus importante que celle entre les voix en anglais et en français. Il illustre également que la différence de l'évaluation des langues pour l'homme et pour la femme ne se présente pas de la même façon. Si, dans l'absolu, la voix de la femme est évaluée d'une façon plus élevée, elle l'est surtout en français tandis que l'homme rattrape cet avantage perceptif pour les caractéristiques dynamique, qualités d'un leader, caractère et ambition - par rapport à la femme, mais seulement s'il parle en anglais. Par contre, les écarts entre l'évaluation de sa voix en anglais et de sa voix en français sont quasiment constants pour toutes les caractéristiques. 
Graphique 5 : Moyennes des évaluations par enregistrement pour les caractéristiques

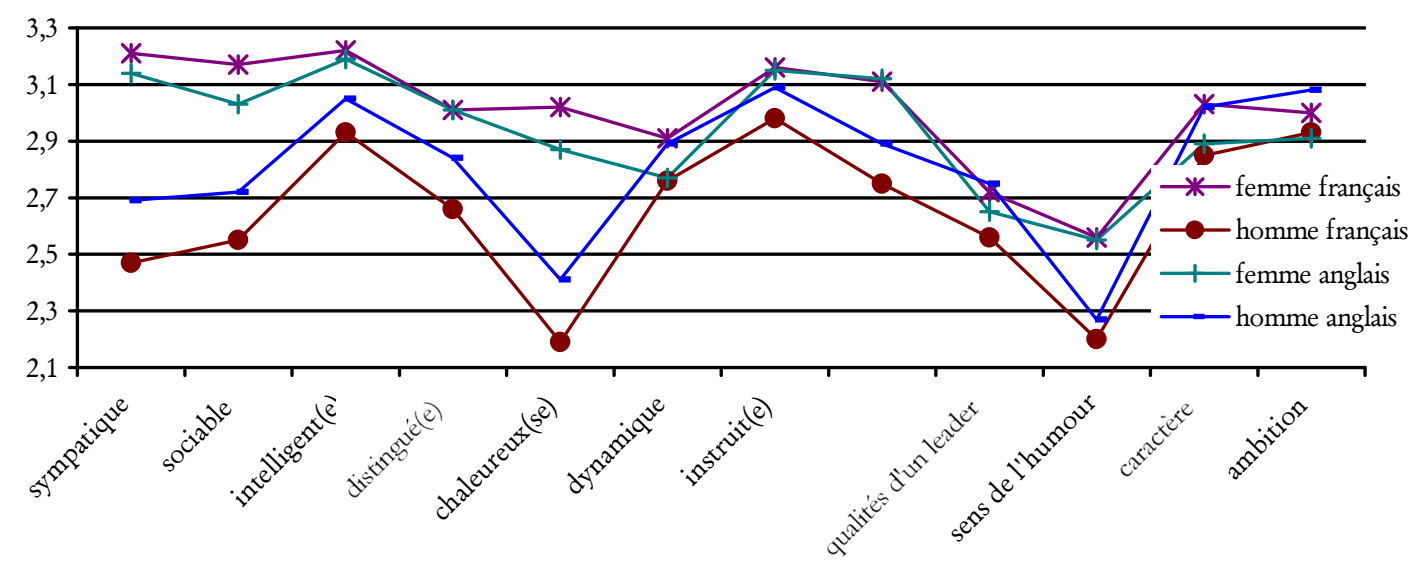

Nous avons exploré avec une autre analyse MANOVA pour savoir si cette interrelation était significative pour les deux entrées de la variable sexe aux deux niveaux de la variable langue (anglais et français) tout comme nous l'avons fait pour les deux entrées linguistiques dans la section précédente. Nous avons donc déjà décrit les effets principaux de ces résultats, mais examinons les effets simples de la langue pour la voix masculine et pour la voix féminine afin de voir les différences des langues pour chaque sexe séparément.

Les résultats indiquent une interaction significative pour la voix féminine et le français pour les traits suivants : sociable, chaleureux, dynamique, caractère et ambition, mais la variance expliquée ne dépasse pas $3 \%$. L'interaction entre la langue et le sexe est par contre significative pour tous les traits lorsque la voix de l'homme est concernée et les moyennes de toutes les caractéristiques sont plus élevées lorsqu'il parle en anglais. Cette variance expliquée ne dépasse pas les $5 \%$. Le facteur statut est non significatif pour la voix féminine; la différence entre les moyennes obtenues pour sa voix en français et en anglais est trop petite. Pour le facteur solidarité, tout comme pour le facteur compétence-action, ces différences sont significatives en faveur de sa voix en français. La voix de l'homme montre des différences significatives pour les trois facteurs, en faveur de sa voix en anglais.

Ces analyses montrent donc encore une fois que la combinaison du sexe et de la langue du locuteur a un effet différencié sur la perception. Nous avons déjà souligné que le fait que ce soit une femme ou un homme qui parle a son importance, et si elle ou il parle l'anglais ou le français également puisque l'évaluation des caractéristiques changera en conséquence, mais nous pouvons dorénavant non seulement affirmer dans quelle combinaison les évaluations diffèrent significativement (pour la voix féminine, c'est le français et pour la voix masculine, l'anglais), mais aussi que la différence entre la femme qui parle en français va être plus importante en comparaison de celle de l'homme qui parle en français que si les deux parlent en anglais. Le graphique suivant illustre ces résultats avec l'exemple du trait chaleureux.

Graphique 6 : Moyennes pour le trait chaleureux

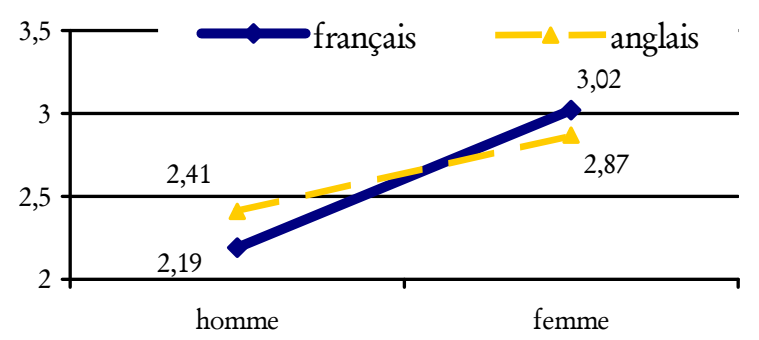


Ainsi, lorsque la voix du locuteur est anglaise, la moyenne pour la femme est plus élevée que celle pour l'homme $(\mathrm{F}=96,62 ; \mathrm{ddl}=1 / 601 ; \mathrm{p}<, 000)$. Lorsque les voix sont en français, la moyenne pour la voix féminine est également plus élevée $(\mathrm{F}=298,16 ; \mathrm{ddl}=1 / 601 ; \mathrm{p}<, 000)$, mais l'ampleur de la différence est plus grande. De plus, le rapport entre les deux langues est inversé selon le sexe du locuteur. La voix en français obtient une moyenne plus élevée que la voix en anglais lorsque la femme parle - tandis que c'est la voix en anglais qui l'emporte lorsque l'homme parle.

En résumé, la différence entre l'évaluation du français et de l'anglais est plus importante lorsque la voix est féminine que lorsqu'elle est masculine. Le facteur compétence-action est coté davantage lorsque la voix est masculine et en anglais, tandis que les autres caractéristiques et facteurs obtiennent une meilleure évaluation pour la femme parlant le français. Alors, si le statut a un genre, il est masculin surtout lorsque cette notion de statut renferme des caractéristiques du facteur compétence.

\subsection{Dimension historique : retour sur les études antérieures}

Pour rendre plus claire cette interaction et ses éventuelles conséquences, nous avons repris l'analyse et la présentation de résultats de quelques études antérieures en utilisant la comparaison des moyennes avec les valeurs des test $t$ et en présentant les valeurs du test sans les moyennes (voir Lambert et autres, 1960: 4647 et Genesee et Holobow, 1989 : 20, 28, 30-32 ). Afin de mieux comparer nos résultats à ceux obtenus autrefois, nous avons comparé les moyennes de la voix de l'homme seulement, en comparant sa voix en anglais et sa voix en français et les moyennes de la voix de la femme en anglais et en français comme s'il s'agissait de deux échantillons indépendants :

Tableau 3 : Valeurs significatives du test $t$ pour les évaluations de voix parlant en français et en anglais selon le sexe (deux échantillons)

\begin{tabular}{|l|c|c|c|c|c|c|}
\multicolumn{1}{c}{} & \multicolumn{3}{c|}{ Voix d'homme } & \multicolumn{3}{c|}{ Voix de femme } \\
\hline \multicolumn{1}{c|}{ traits } & français & anglais & ddl & français & anglais & ddl \\
\hline sympathique & - & $5,147 * * *$ & 606 & - & - & 604 \\
\hline sociable & - & $3,914 * * *$ & 607 & $3,494 * *$ & - & 607 \\
\hline intelligent(e) & - & $3,481 * *$ & 600 & - & - & 601 \\
\hline distingué(e) & - & $4,092 * * *$ & 598 & - & - & 600 \\
\hline chaleureux(se) & - & $4,956^{* * *}$ & 602 & $3,343 * *$ & - & 600 \\
\hline dynamique & - & $2,990 * *$ & 602 & $3,450 * *$ & - & 607 \\
\hline instruit(e) & - & $3,570 * * *$ & 601 & - & - & 600 \\
\hline fiable & - & $3,685 * * *$ & 595 & - & - & 595 \\
\hline qualités d'un leader & - & $3,965 * * *$ & 602 & - & - & 601 \\
\hline sens de l'humour & - & - & 604 & - & - & 598 \\
\hline caractère & - & $4,300 * * *$ & 602 & $4,187 * * *$ & - & 598 \\
\hline ambition & - & $4,113 * * *$ & 597 & $2,629 * *$ & - & 602 \\
\hline
\end{tabular}

Ainsi, en contrôlant pour le sexe des voix, il devient évident que les moyennes générales plus hautes pour le français proviennent d'une forte évaluation de la femme dans cette langue - puisque l'homme est évalué plus fortement sur quasiment toutes les caractéristiques quand il parle en anglais. Donc, si nous nous limitions dans notre analyse aux seules voix de l'homme, nos résultats montreraient ce à quoi les autres études faisaient référence : une évaluation systématiquement plus forte de l'anglais.

\section{Bref retour aux résultats de 1960 et de 1989}

Ce qui aurait pu être une simple limite méthodologique se révèle néanmoins plus lourd de conséquences, notamment à cause des interprétations fournies. Lambert et autres (1960) concluent leur étude comme suit :

«The results indicate that English Ss [Speakers] evaluate the English guises more favourably on most traits. French Ss not only evaluate the English guises more favourably than French guises, but their evaluations of French guises are reliably 
less favourable than those of English Ss. This finding is interpreted as evidence for a minority group reaction on the part of the French sample » (p. 50-51).

Le résultat indique donc que les anglophones tout comme les francophones évaluent plus favorablement les locuteurs lorsqu'ils parlent en anglais. Les chercheurs interprètent ce fait comme une réaction d'un groupe minoritaire. Avant tout, pour avoir une réaction d'un groupe minoritaire, les francophones auraient dû être minoritaires, c'est-à-dire présenter un nombre démographique moins fort qu'un autre groupe montréalais - ce qui n'était pas le cas dans les années 60. Cette interprétation laisse donc supposer une confusion entre différentes formes de minorisation telles que nous les connaissons aujourd'hui : démographique, symbolique, économique, politique, etc. La confusion serait donc celle qui existe entre «minorité » et «domination»: une minorité est numériquement inférieure à un autre groupe, une domination est par contre matérielle ou symbolique et peut être tout aussi bien exercée par une majorité ou une minorité numérique. Le choix du mot «minoritaire» de la part des chercheurs dans leur interprétation des résultats ne peut donc pas renvoyer à une minorisation numérique, mais doit forcément faire référence à un autre fait de domination. Les chercheurs examinent en effet la possibilité d'une interprétation socio-économique de leurs résultats, mais finissent par rejeter l'hypothèse voulant qu'une motivation socio-économique ou symbolique puisse expliquer certaines différences dans les perceptions. C'est-à-dire que lors d'une autre tâche, les étudiants devaient attribuer des catégories d'emploi (professionnelles ou non professionnelles) aux extraits des voix en anglais et en français. Tous les étudiants évaluaient en moyenne les voix en anglais comme étant plus professionnelles mais, puisque les étudiants du collège francophone le faisaient d'une façon moindre que les étudiants de l'université McGill, les auteurs trouvaient que ceci « ...argues against an interpretation in terms opf differences in perceived status of the two groups. » (Lambert et al. 1960 : 47). Or, déjà lors de la publication de l'étude originale, Tajfel (1959) faisait référence à une association socio-économique qui manque dans l'interprétation donnée par Lambert et ses collègues : « ... the fact is established that the classification onto French and English is correlated with socio-economic status, both objectively and subjectively » (p. $88)$.

Il semble évident que, à l'époque encore davantage qu'aujourd'hui, ces deux langues, encore toutes les deux officielles au Québec dans les années 60, avaient une connotation nettement différente quant à leur prestige ou à leur attrait socio-économique. D'ailleurs, le choix de Lambert et de ses collègues de nommer leur résultat une réaction de «minoritaire» y renvoie directement comme nous l'avons fait remarquer. Mais encore aujourd'hui l'évaluation de l'anglais à Montréal est une évaluation d'une langue qui jouit non seulement d'un statut de langue officielle dans le pays dont le Québec est une province, mais aussi d'une langue de renommée internationalement (voire globalement) véhiculaire. Mais l'anglais est également une langue avec une histoire de presque 300 ans au Québec - une langue associée à la victoire d'abord militaire et ensuite socio-économique des anglophones sur le territoire (Blair, 2005; Dickinson et Young, 1995). Rien d'étonnant alors que cette langue soit perçue davantage comme étant prestigieuse et, surtout, comme langue de statut. Ignorer ce fait est une chose, en démentir l'effet en est une autre.

Trente ans plus tard, une étudiante de l'Université McGill à Montréal choisissait de refaire pour sa thèse de doctorat (sans copier aveuglément tous les aspects de la première étude) la même recherche à Montréal. Les résultats, publiés dans Genesee et Holobow (1989), ressemblent grosso modo à ceux présentés dans l'étude originale :

\section{"A comparison of these results with Lambert et al.'s (1960) results reveals that there is very little difference between our francophone respondents' ratings and Lambert's francophone respondents' ratings on the status traits - both groups of respondents evaluated the Canadian English guises more favourably than the Quebec French guises on all status traits » (p. 27).}

Le résultat est donc resté le même : pour le statut, ce sont les voix en anglais qui sont évaluées d'une façon plus favorable par les deux groupes linguistiques. À partir de ce constat, l'interprétation d'une réaction psychologique face à un groupe dominant semble confirmée par Genesee et Holobow, mais ils vont plus loin dans leur interprétation des mêmes faits: «The status results are interpreted in terms of a 
generalized psychological stereotype of French Canadians that is relatively immune to objective evidence » (p. 17). Il n’y a pas de précision quant au contenu de cette « évidence objective » qui serait ignorée par les francophones. Il n'empêche que, reprise dans la littérature scientifique, cette interprétation d'une réaction minoritaire sans évidence objective est devenue entre autres « une forme de haine de soi » (Bourhis et Lepicq, 1993 : 362) de la part des francophones. D’une dominance interprétée en « réaction minoritaire », nous passons par un «stéréotype psychologique généralisé » «imperméable à l'évidence objective » pour aboutir à une « forme de haine de soi ».

Dans l'étude présentée ici, nous avons choisi d'utiliser la même méthodologie ainsi que (pour l'effet des voix) les mêmes analyses statistiques afin de pouvoir comparer certains des résultats, surtout l'effet des évaluations lorsqu'on ne considère que les voix de l'homme. Le résultat est éloquent: l'homme est systématiquement mieux évalué en anglais qu'en français. On pourrait attribuer ce résultat au seul effet de la voix d'une personne en particulier, mais cette supposition se révèle injustifiée lorsqu'on analyse les voix de la femme, qui, généralement, se trouvent mieux évaluées. Ainsi, nous pouvons confirmer le résultat des autres études, mais seulement pour la voix d'homme. Lorsqu'on introduit la voix d'une femme, ce résultat ne tient plus et les interprétations (qui généralisent du même coup le comportement de quelques étudiants à l'échelle du Québec) semblent exagérées, surtout lorsqu'on considère que ce sont tous les Montréalais qui évaluent plus fortement l'anglais (voir entre autres les moyennes du graphique 1b). La réaction minoritaire qui était à la base de l'interprétation de Lambert et autres et qui semblait se confirmer avec le temps (Genesee et Holobow, 1989), ne se retrouve qu'en limitant l'échantillon d'une façon importante. Seul un échantillon aléatoire aurait pu confirmer ce fait.

Il s'avère ainsi qu'une simple omission (celle de la voix de la femme pour l'évaluation), peu importe qu'elle soit due au climat social de l'époque ou à une attitude linguistique particulière, peut générer des interprétations qui, en plus de sembler aujourd'hui abusives, ont aussi eu un impact considérable sur les études québécoises à travers le temps.

\section{Conclusion}

Notre perception diffère selon les voix que nous entendons. Les caractéristiques des voix ne sont pas anodines dans le jugement que nous portons sur les locuteurs. Il importe de savoir si notre interlocuteur est un homme ou une femme et quelle langue cette personne parle. L'évaluation des langues diffère selon le genre des locuteurs, c'est-à-dire que la perception des langues n'est pas asexuée. En d'autres mots : l'anglais est mieux évalué lorsqu'un homme parle, tandis que c'est le français lorsque c'est une femme. Une telle différence dans la perception concerne surtout les traits associés à une « compétence-action »en faveur de l'anglais et en faveur de la voix de l'homme. S'agit-il moins d'un stéréotype que, d'un côté de la reconnaissance d'un statut international supérieur de l'anglais ou de son statut comme langue du commerce par rapport au français et, de l'autre, d'une perception plus arrêtée sur le statut masculin? Davantage de recherches sont nécessaires afin de mieux comprendre cette inversion des perceptions. Évidemment, ce ne sont pas les voix qui créent réellement une différence dans la perception des gens. Il s'agit plutôt d'une inférence apprise qui guide nos associations en fonction de différentes caractéristiques des voix. Dans cette étude, nous avons pu en explorer deux : le sexe et la langue. Ce sont les seules caractéristiques de voix que nous avons analysées, puisque la méthodologie des faux-couples devait justement déguiser des indices qui peuvent révéler l'identité de la personne qui parle. Il y a également lieu de consolider les résultats de cette recherche avec un plus grand nombre de personnes bilingues.

Tout comme les études menées à Montréal sur le même sujet avant celle-ci, nous avons trouvé une certaine association du statut et de la compétence en faveur de l'anglais et en faveur de la voix masculine. L'évaluation plus forte des traits de compétence constitue en fait une preuve indirecte que la perception d'une langue mesurée à l'échelle d'une population peut refléter directement une réalité externe. Nous avons déjà argumenté dans ce sens en 2001 à partir de données sur l'association entre la langue majoritaire et le degré de richesse dans des quartiers de Montréal (Laur, 2001). Réitérons également que cette association fait preuve d'une certaine continuité et stabilité dans les perceptions au cours de presque un demi-siècle. 
La méthodologie des faux-couples reste une invention ingénieuse pour analyser les attitudes et les perceptions d'une manière indirecte. Néanmoins, cette méthodologie ne permet de généraliser les résultats qu'à la population interrogée (ou, lorsqu'elle est représentative, à la population cible) et porte en elle une limite majeure dont il faut rendre compte : le contraste des paires de traits « couplés ». En prenant pour exemple le genre, cette étude montre qu'un seul trait (omis ou ajouté) peut avoir des conséquences saisissantes sur l'évaluation des langues ou de variétés de langue, et donc sur la connaissance que ces études peuvent nous apporter sur leurs interrelations sociales.

\section{Références bibliographiques}

Armstrong N., Bauvois C. et K. Beeching (eds.). (2001). La langue française au féminin. Paris : L'Harmattan.

Blair, L. (2005). Les Anglos. La face cachée de Québec. Tome I. 1608-1850. Québec: Commission de la capitale nationale du Québec.

Blair, W., G. Jarvis et R.E. Petty (1996). The need to evaluate. Journal of Personality and Social Psychology, 70/1, 172-194.

Bourhis, R.Y. et D. Lepicq (1993). Quebecois French and Language Issues in Quebec. Dans: Posner, R. et J.N. Green (dirs), Trends in Romance Linguistics and Philology, Vol.5 : Bilingualism and Linguistic Conflict in Romance. Berlin: de Gruyter, 345-381.

Calvet, L-J. (1999). La guerre des langues et les politiques linguistiques. Paris :Hachette.

Dickinson, J. A. et B. Young (1995). Brève histoire socio-économique du Québec, Nouvelle édition mise à jour. Sillery : Les éditions du Septentrion.

Dixon, J. A., B. Maltoney et R. Cocks (2002). Accents of guilt. Effects of regional accent, race, and crime type on attributions of guilt. Journal of Language and Social Psychology, 21/2, 162-168.

Genesee, F. et N.E. Holobow (1989). Change and stability in intergroup perceptions. Journal of Language and Social Psychology, 8/1, 17-38.

Giles, H. et E. B. Ryan (1982). Prolegomena for developing a social psychological theory of language attitudes. Dans E.B. Ryan et H. Giles (dirs) Attitudes Towards Language Variation: Social and Applied Contexts, London: Edward Arnold, 208-223.

Kimura, D. (1999). Sex and Cognition, Cambridge: MIT Press.

Labelle, M. http://wwwens.uqac.ca/ flabelle/socio/genre.htm. Site consulté le 27-12-07.

Labov, W. (1990). The interaction of sex and social class in the course of linguistic change. Language Variation and Change, 2, 205-254.

Labov, W., S. Ash et Ch. Boberg (2004). Atlas of North American English. Phonetics, Phonology and Sound Change, Berlin: Mouton de Gruyter.

Lambert, W. E., R.C. Hodgson, R. Gardner et S. Fillenbaum (1960). Evaluational reactions to spoken languages. Journal of Abnormal and Social Psychology, 60, 44-51.

Laur, E. (2001). Perceptions linguistiques à Montréal. Thèse de doctorat, Université de Montréal.

Laur, E. (2002). La qualité, le statut et la perception du français au Québec. Revue d'aménagement linguistique, Hors série, 147-165.

Laur, E. (à paraître). Contribution à l'étude des attitudes linguistiques. La méthodologie des faux-couples revisitée. Langue et Société, Note méthodologique 46.

Luc, É. (1989). Attentes d'efficacité, attributions et difficulté perçue dans des situations de communication orale chez les francophones et les anglophones. Thèse de doctorat, Université de Montréal.

Osgood, Ch. E. (1964). Semantic differential technique in the comparative study of cultures. American Anthropologist, 66/3, 171-200.

Preston, M.S. (1963). Evaluational Reactions to English, Canadian French and European French Voices. Mémoire de maîtrise, Université McGill. 
Tajfel, H. (1959). A note on Lambert's 'evaluational reactions to spoken languages'. Canadian Journal of Psychology,13, 86-92.

Trudgill, P. (1972). Sex, covert prestige and linguistic change in the urban British English of Norwich. Language in Society, 1, 179-195.

Zahn, Ch.J. et R. Hopper (1985). Measuring language attitudes: The speech evaluation instrument, Journal of Language and Social Psychology, 4/2, 113-123.

${ }^{1}$ Les extraits de voix incluent un homme et une femme bilingue ayant lu le même court extrait d'une œuvre littéraire montréalaise en français et un autre extrait en anglais. L'authenticité du bilinguisme de ces enregistrements a été testée par le biais d'un prétest.

${ }^{2}$ Nous avons procédé à une enquête téléphonique auprès de 610 répondants, représentatifs de la population de l'île de Montréal. Cette base de sondage provient d'un échantillon qui est généré par un logiciel d'échantillonnage aléatoire de numéros de téléphone. Le répondant est sélectionné par la grille de Kish. Le taux de réponse est de 51,3\%. Les entrevues ont été réalisées du 17 juin au 4 juillet 2004.

${ }^{3}$ L'interaction entre la langue et le sexe des voix des locuteurs par (1) traits : sympathique ( $\mathrm{F}=27,75$; $\mathrm{ddl}=1 / 601 ; \mathrm{p}<, 000 ; \mathrm{y}^{2}$ eta carré=,044), sociable $\left(\mathrm{F}=26,85 ; \mathrm{ddl}=1 / 605 ; \mathrm{p}<, 000 ; \mathrm{\eta}^{2}=, 042\right)$, intelligent $\left(\mathrm{F}=11,64 ; \mathrm{ddl}=1 / 596 ; \mathrm{p}<, 001 ; \mathrm{n}^{2}=, 019\right)$, distingué $\left(\mathrm{F}=8,27 ; \mathrm{ddl}=1 / 595 ; \mathrm{p}<, 004 ; \mathrm{n}^{2}=, 014\right)$, chaleureux $\left(\mathrm{F}=34,42 ; \mathrm{ddl}=1 / 601 ; \mathrm{p}<, 000 ; \mathrm{n}^{2}=, 054\right)$, dynamique $\left(\mathrm{F}=20,15 ; \mathrm{ddl}=1 / 602 ; \mathrm{p}<, 000 ; \mathrm{p}^{2}=, 032\right)$, instruit $\left(\mathrm{F}=7,06 ; \mathrm{ddl}=1 / 596 ; \mathrm{p}<, 008 ; \mathrm{n}^{2}=, 012\right)$, fiable $\left(\mathrm{F}=6,71 ; \mathrm{ddl}=1 / 588 ; \mathrm{p}<, 010 ; \mathrm{y}^{2}=, 011\right)$, qualités d'un leader $\left(\mathrm{F}=15,54 ; \mathrm{ddl}=1 / 596 ; \mathrm{p}<, 000 ; \mathrm{y}^{2}=, 025\right)$, sens de l'humour $\left(\mathrm{F}=1,49 ; \mathrm{ddl}=1 / 594 ; \mathrm{p}>, 223 \mathrm{~ns} ; \mathrm{\eta}^{2}=, 002\right)$, caractère $\left(\mathrm{F}=36,98 ; \mathrm{ddl}=1 / 594 ; \mathrm{p}<, 000 ; \mathrm{n}^{2}=, 059\right)$, ambition $\left(\mathrm{F}=23,31 ; \mathrm{ddl}=1 / 591 ; \mathrm{p}<, 000 ; \mathrm{n}^{2}=, 038\right)$ et $\operatorname{par}(2)$ facteurs : statut $\left(\mathrm{F}=13,22 ; \mathrm{ddl}=1 / 576 ; \mathrm{p}<, 000 ; \mathrm{n}^{2}=, 022\right)$, solidarité $(\mathrm{F}=27,54 ;$ ddl $=1 / 587 ; \mathrm{p}<, 000$; $\left.\mathrm{y}^{2}=, 045\right)$, compétence-action $\left(\mathrm{F}=37,27 ; \mathrm{ddl}=1 / 575 ; \mathrm{p}<, 000 ; \mathrm{y}^{2}=, 061\right)$. 